\title{
Utility of $p$-Acetamidobenzoyl Prop-2-enoic Acid in the Synthesis of New $\alpha$-Amino Acids and Using Them as Building Blocks in Heterocyclic Synthesis
}

\author{
M.A.El-Hashash, S.A. Rizk ${ }^{*}$ and M.M. Aburzeza* \\ Chemistry Department, Faculty of Science, Ain Shams \\ University, Cairo, Egypt and *Chemistry Department, Faculty \\ of Science, Elmargeb University, Zliten, Libya.
}

\begin{abstract}
7 HE PRESENT work deals with the generation and synthesis of different unnatural amino acid derivatives via treatment of 3-(4acetamidobenzoyl)-prop-2-enoic acid with 5-aryl-2- amino 1, 3, 4 thiadiazole, 3, 5-dimethyl pyrazole and barbituric acid to afford the product of conjugate addition acids (1) respectively. Additionally, the adduct 1 are used as key starting materials to synthesize some heterocycles include pyridazene, furanone and oxazine derivatives. The antimicrobial screening of some of the synthesized compounds was done using the agar diffusion assay
\end{abstract}

Keywords: 3-Acetamidobenzoyl prop-2-enoic acid, Pyrazole thiadiazole , Phthalimide , Barbituric acid, Furanone, Pyridazine and Oxazine.

Amino acids are the smallest unit of protein and are useful components in a variety of metabolisms. There are more than advantages of taking amino acids dietary supplements come from their many useful biological activities. In vitro data ${ }^{(1)}$ about amino acids include muscle protein maintenance, potentiation of immune function, affecting neuronal activities in the brain, tissue repair acceleration, protecting liver from toxic agents, pain relief effect, lowering blood pressure, modulating cholesterol metabolism, stimulating insulin of growth hormone secretion and so on. It is important to be aware that they are part of complex pathway and biological systems. Amino acids have proven to play a significant role in the synthesis of novel drug candidate with the use of nonproteinogenic and unnatural amino acids ${ }^{(2-9)}$. Thus, we reported the reactions of 3( $p$-acetamidobenzoyl) 2-(2-amino 5-aryl thiadiazole) propionic acids 1 with $\mathrm{N}_{2} \mathrm{H}_{4}$, phenylhydrazine, hydroxyl amine and acetic anhydride to give the corresponding pyridazinone, oxazinone and furanone derivative, respectively aiming to afford some interesting heterocyclic compounds with non-mixing and mixing system .

\footnotetext{
\#Corresponding author: Samehrizk2006@gmail.com .
} 


\section{Results and Discussion}

When 3 ( $p$-acetamidobenzoyl) prop -2- enoic acid was allowed to react with 2-amino 5-aryl thiadiazole derivatives, it afforded the product of conjugate addition , 3 ( $p$-acetamidobenzoyl) -2-(5-aryl 2-thiadiazolyl amino) propanoic acids as $\alpha$-amino acid types that differ in biological activity by differing the aryl groups (Table 1) (Scheme1). IR spectra of acids 1 exhibit strong absorption at $(1695-1630) \mathrm{cm}^{-1} \mathrm{CO}$ for acid and ketone groups in addition to $v \mathrm{CO}$ in case of $1_{c}$ and $1_{f}$ at $(1770-1712)$ and (1615) corresponding to phthalimido and barbiturate moieties, respectively.

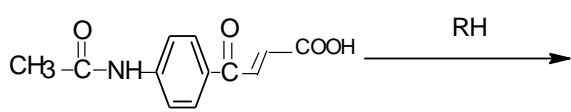<smiles>Nc1nnc(-c2ccccc2)s1</smiles><smiles>Nc1nnc(C=Cc2ccccc2)s1</smiles><smiles>Nc1nnc(-c2ccc(Cl)cc2)s1</smiles>

b<smiles>CC1=NN=CC1C</smiles><smiles>CC(=O)Nc1ccc(C(=O)CC(=O)O)cc1</smiles>

1<smiles>Nc1nnc(Cn2oc3ccccc3c2=O)s1</smiles>

C<smiles>CN1C(=O)CC(=O)NC1=O</smiles>

Moreover, reactivity of $\mathrm{C}_{2}$ in 3 -aroyl propio-2-enoic acids ${ }^{(10-11)}$ allows azamichael addition by 2ry amine, e.g 4,5-dimethyl pyrazole and barbaturic acid . The preference for nucleophilic addition at $C_{2}$ is discussed in terms of a) stability of resonance forms of the acrylic starting material. The relative stabilities of the alternative structures of the primary zwitter ionic adduct indicate that the negative charge on the enolate moiety resulting from addition at $\mathrm{C}_{2}$ to be more delocalized than $\mathrm{C}_{3}$ as below:
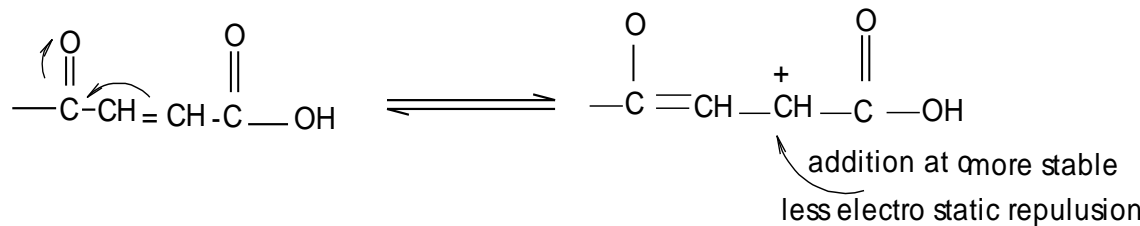<smiles>CC(=O)/C=C/C(=O)O</smiles><smiles>CC(=O)[CH+]C=C([O-])O</smiles>

less stable more electro static repulusio 
b) An electronic influence of the $p$-acetamidophenyl group on C3 may be present, the activating acetamido group in $p$-position may be made to prefer attack at C3. c) steric hindrance of $p$-acetamidophenyl group to addition at $\mathrm{C} 3$ may also play a role.

Since the entering nucleophile attacks $\mathrm{C} 2$ at expence of $\mathrm{C} 3$, so reasons 1 and 3 outweigh reason 2 , in the preference of addition of an amino group to $\mathrm{C} 2$ of acids 1 . The acids 1 are confirmed by spectroscopic tools, EIMS for acids 1 are 1a $m / z 377\left(\mathrm{M}^{+}-\mathrm{CO}+\mathrm{H}_{2}\right), 1_{\mathrm{b}} \mathrm{m} / \mathrm{z} 358\left(\mathrm{M}^{+}-\left(\mathrm{CO}_{2}+\mathrm{CH}_{2}=\mathrm{CO}\right), 1 \mathrm{c} m / z ; 446\right.$ $\left(\mathrm{M}^{+}-\mathrm{CO}_{2}\right)$, 1e $m / z: 307\left(\mathrm{M}^{+}-\mathrm{H}_{2} \mathrm{O}\right)$. The explanation of one ${ }^{1} \mathrm{HNMR}$ spectrum for acids 1 ,e.g., $1_{\mathrm{b}}$ in DMSO exhibits signals at $2.5\left(\mathrm{~s}, 3 \mathrm{H}, \mathrm{CH}_{3} \mathrm{CO}\right), 3.4(2 \mathrm{dd}$, $\mathrm{CH}_{2-} \mathrm{C}=\mathrm{O} \mathrm{J}=15.2, \mathrm{~J}=7.7$ ) (diastereotopic protons) adjacent to ketonic group are non equivalent and each proton appears as douplet (4 lines, $\mathrm{dd}, \mathrm{J}=15.2$ ), each line couples with methine proton $\mathrm{J}=7.7$ and gives two douplet of douplets (8 lines, $2 \mathrm{dd})$ ), 4.2 (dd,CH-COOH,methine proton) 6.7 (s,NH),7.6-8.1 (m,8H,ArH) $\mathrm{ArH}), 8.2 \quad(\mathrm{~s}, 1 \mathrm{H}, \mathrm{COOH}), 8.6 \quad(\mathrm{~s}, 1 \mathrm{H}, \mathrm{C}=\mathrm{O}-\mathrm{NH})$. It was reported ${ }^{(12)}$ that the pyridazinone substituted 1,3,4- thiadiazolene were shown to be fungicidally active and their activity was influenced by the nature of the substituents. Thus, when acids 1 a-c were allowed to react with hydrazine hydrate in boiling ethanol, they afforded 6- acetanilido- 4 (5-aryl-2-amino 1,3,4 thiadiazole) 2,3 dihydro $3(2 \mathrm{H})$ pyridazinones $\left(2_{\mathrm{a}-\mathrm{c}}\right)$, not tetrahydropyridazinones ${ }^{(20)}$. The authors can explain auto-oxidation of initially formed tetrahydropyridazinones results dihydropyridazinone 2 due to the presence of activating aromatic moieties in position 4 enhancing the dehydrogenation of initially formed tetrahydropyridazinones, formation of extra conjugating system afforded more thermodynamic stable 2 . IR spectra of 2 a-c reveal strong absorption bands at $1630 \mathrm{~cm}^{-1}$ for $v C O$ cyclic carboxamide and $(3260-3270)$ for $v \mathrm{NH}$ whatever $v$ $\mathrm{CO}$ in phthalimido moiety in 2. EIMS for $2_{\mathrm{a}} m / z: 404\left(\mathrm{M}^{+}\right), 2_{\mathrm{b}} m / z: 403\left(\mathrm{M}^{+}-\mathrm{Cl}\right)$, $2_{\mathrm{c}} \mathrm{m} / \mathrm{z}: 426\left(\mathrm{M}^{+}-\left(\mathrm{CH}_{3}+\mathrm{H}_{2}\right)\right.$. The explanation of one ${ }^{1} \mathrm{HNMR}$ spectrum for Compounds 2, e.g., 2 a revealed singlet at 2.5 assigned to methyl group, multiplet at $7.4-7.8$ assigned for aromatic protons, singlet at $7.2 \mathrm{ppm}$ for protons of pyridazinone, singlet at 10.2 assingned for two acidic protons of acetamido and pyridazinone moieties and singlet broad band at $6.5 \mathrm{ppm}$ assigned for $\mathrm{NH}$ of thiadiazole moiety. Substituted pyrazole and barbiturate moieties constitute an important class of compounds in the field of agricultural and medicinal chemistry because of their broad spectrum biological activities ${ }^{(13)}$. Since, the combination of two or more heterocyclic and nonheterocyclic ${ }^{(14)}$ systems enhances the biological profile more fold than their parent nuclei, so we considered to synthesize ; the pyridazinone has pyrazolyl and / or barbituryl groups in position 4 . Treatment of the acids $1_{\mathrm{e}, \mathrm{f}}$ with $\mathrm{N}_{2} \mathrm{H}_{4}$ in boiling ethanol, afforded 6-( $p$-acetamido phenyl)-4 (3,5-dimethyl pyrazolyl) and / or 2,4 dihydroxy pyrimidinyl 6-one) $2,3,4,5$ tetrahydro $3(2 \mathrm{H})$ pyridazinone $\left(2_{\mathrm{d}, \mathrm{e}}\right)$. IR spectra of $2_{\mathrm{d}, \mathrm{e}}$ reveal strong absorption bonds at $\left(1640 \mathrm{~cm}^{-1}\right)$ for $v \mathrm{CO}$ of pyridazinone, EIMS for $2_{\mathrm{d}} \mathrm{m} / \mathrm{z}: 325\left(\mathrm{M}^{+}\right)$. Recently ${ }^{(15)} 2(3 \mathrm{H})$ furanone exhibit rich photochemistry, furthermore, due to their common occurrence in nature, oxygen containing heterocyclic are frequent and important target, for synthesis 
either as final products or as useful synthetic intermediate. Thus, when acids 1 was allowed to react with Ac2O on heating water bath for $1 \mathrm{hr}$, they have $5(\mathrm{p}$ acetamido phenyl )-3-(5-aryl-2-1,3,4 thiadiazolylamino )-2- $(3 \mathrm{H})$ furanone $(3 \mathrm{a}-\mathrm{b})$ and/or 5( $p$-acetamidophenyl ) -3 (2,4,6 dihydroxy pyrimidinyl -6-on )-2- $(3 \mathrm{H})$ furanone $\left(3_{\mathrm{f}}\right)$. Their structures $3_{\mathrm{a}, \mathrm{b}, \mathrm{d}}$ were inferred from IR spectra which exhibit strong absorption bands at $\left(1767-1755 \mathrm{~cm}^{-1}\right)$ attributable to $v \mathrm{CO}$ (lactonic) in addition to other carbonyl of acetamido gp at $1693 \mathrm{~cm}^{-1}$. The ${ }^{1} \mathrm{H}$ NMR spectrum of compound 3a in DMSO exhibits signals at $\delta 2,1\left(\mathrm{~s}, 3 \mathrm{H}, \mathrm{CH}_{3}, \mathrm{CO}\right.$ ), 4, (dd $1 \mathrm{H},-\mathrm{C} \underline{\mathrm{H}}-\mathrm{NH} \mathrm{J}=8.5)$ 7.5-7.9 (m,9H of $\mathrm{Ar}$ and $1 \mathrm{H}$ of furanone moieties), 6.7 $(\mathrm{d}, 1 \mathrm{H}, \mathrm{NH}, \mathrm{J}=8.5), 12.7(\mathrm{~s}, 1 \mathrm{H}-\mathrm{C}=\mathrm{O}-\mathrm{N} \underline{\mathrm{H}})$ acidic protons exchangeable in $\mathrm{D}_{2} \mathrm{O}$. EIMS for compound $3_{\mathrm{f}} \mathrm{m} / \mathrm{z}: 343\left(\mathrm{M}^{+}\right)$. But interaction of acids $1_{\mathrm{c}, \mathrm{e}}$ with acetic anhydride afforded ketones 4 . Formation of ketones 4 is due to the decarboxylation of acid $1_{\mathrm{c}, \mathrm{e}}$ via its heating at high temperature ${ }^{(16)}$ presented as follow.

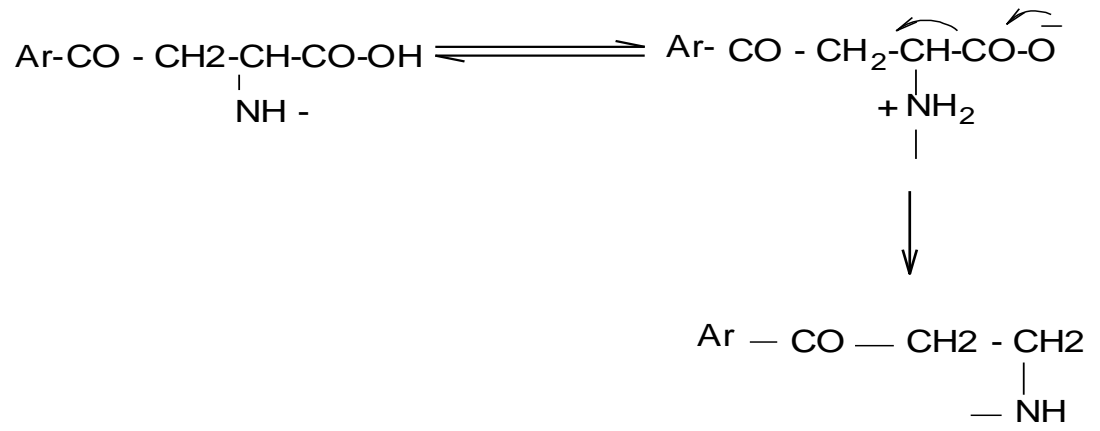

Here, the authors offer a speculation that stability of a zwitter ionic intermediate facile decarboxylation takes place in acids $1_{c, e}$. The presence of phthalimido and pyrazolyl groups which contain basic nitrogen enhances formation of a zwitter intermediate. IR spectra of 4 revealed absorption bands at ( $1706 \mathrm{~cm}^{-1}$ ) corresponding to $v \mathrm{CO}$ of ketones. EIMS for compound $4 \mathrm{c}, \mathrm{m} / \mathrm{z}$; $423\left(\mathrm{M}^{+}-\mathrm{CO}\right)$. On the other hand, oxazinone derivatives are an important clan of heterocyclic compounds, since many of their heterocyclic system exhibit biological activity ${ }^{(17)}$. This promoted us to synthesize 1,2 oxazine derivatives incorporating with heterocyclic moieties in the position-5. Thus, the reaction of acids 1 with hydroxyl amine in refluxing pyridine, gave 3- ( $p$-acetamido phenyl)5- (5 aryl-2-amino-1,3,4thiadiazole and lor 2,4-dihydroxy-6-oxo pyrimidin-yl ) 1,2 oxazin -6-one 5. Their structures were inferred from IR spectra which exhibit strong absorption bands at $1712-1703 \mathrm{~cm}^{-1}$ corresponding $v$ CO of azalactone irrespective $\mathrm{CO}$ for phthalimido and pyrimidinone moieties. EIMS for $5 \mathrm{c} \mathrm{m} / \mathrm{z}$ at $271\left(\mathrm{M}^{+}-\left(\mathrm{Co}_{2}+\mathrm{CH}_{2}=\mathrm{C}=\mathrm{O}\right)\right)$. The one of ${ }^{1} \mathrm{H}-\mathrm{NMR}$ for oxazinone derivatives, $5_{\mathrm{f}}$ in DMSO exhibits signals at $2.45\left(\mathrm{~s}, 3 \mathrm{H}_{1} \mathrm{CH}_{3}-\mathrm{C}=\mathrm{O}-\right)$, 7.0-7.4 (dd, $4 \mathrm{H}$, phenyl group) , $8.0(\mathrm{~s}, 1 \mathrm{H}$,oxazin $(\mathrm{H} 4)), 6.9\left(\mathrm{~s}, 2 \mathrm{H},-\mathrm{C}=\mathrm{O}-\mathrm{CH}_{2}-\mathrm{C}=\mathrm{O}-\right.$ in barbiturate $), 8.3$ $(\mathrm{s}, 1 \mathrm{H}$, pyrimidine moiety $1: 1), 9.9(\mathrm{~s}, 1 \mathrm{H},(\mathrm{OH} \mathrm{NH})$ in pyrimidine = barbiturate equilibrium), $12.5(\mathrm{~s}, 1 \mathrm{H},-\mathrm{C}=\mathrm{O}-\mathrm{NH})$ of acetamido moiety. In the present work, treatment of acids 1 with phenyl hydrazine in boiling ethanol yielded 2-phenyl- 4- (5-

Egypt. J. Chem. 54, No.3 (2011) 
[p-chlorophenyl) 1,3,4 thiadiazol-2-amino] and/or 2,4-dihydroxy-6- oxopyrimidinyl ) -6- ( $p$-acetanilido ) $3(2 \mathrm{H})$ pyridazinone 6 . IR spectra reveal strong absorption bands at $\left(1632 \mathrm{~cm}^{-1}\right)$ corresponding to vCO in $6 a$ and $1694-1650 \mathrm{~cm}^{-1}$ corresponding to two vCO of carbonyl of oxopyrimidinyl and pyridazinone moieties, respectively. Pyridazinone derivative $2 \mathrm{a}$ reacts with ethyl acetoacetate in the presence of potassium carbonate in dry acetone ${ }^{(18,19)}$ to give 3(ethoxycarbonyl methoxy)-4- (5phenyl-1,3,4 thiadiazol-2- amino)-6- acetanilido- 4,5- dihydro pyridazine 7. Its IR spectrum reveals strong absorption band for ester group, EIMS for compound 7 $\mathrm{m} / \mathrm{z}$ : 492 corresponding to $\mathrm{M}^{+}$. Hydazinolysis of oxazinone derivative 5a by hydrazine hydrate in boiling ethanol afforded 2-amino- 4( 5-phenyl 1,3,4 thiadiazol 2-amino ) -6- ( $p$-acetamidophenyl) 2,3dihydropyridazin-3-one 8 . IR spectra reveals strong absorption band at $1671 \mathrm{~cm}^{-1}$ corresponding to $v \mathrm{CO}$ of carbonyl of pyridazinone moiety. The ${ }^{1} \mathrm{H}-\mathrm{NMR}$ for compound 8 in DMSO exhibits signals at $2.5(\mathrm{~s}, 3 \mathrm{H}, \mathrm{CH} 3 \mathrm{CO}), 5.9-6.5\left(\mathrm{~m}, 3 \mathrm{H}, \mathrm{NH} 2 \& \mathrm{NH}\right.$, which disappear in $\left.\mathrm{D}_{2} \mathrm{O}\right), 6.9-7.6$ $(\mathrm{m}, 9 \mathrm{H}$, Aromatic protons), $8.1(\mathrm{~s}, 1 \mathrm{H}$, proton of pyridazine moiety), 12.5 (s,1H,CO$\mathrm{NH})$ which disappears by $\mathrm{D}_{2} \mathrm{O}$.

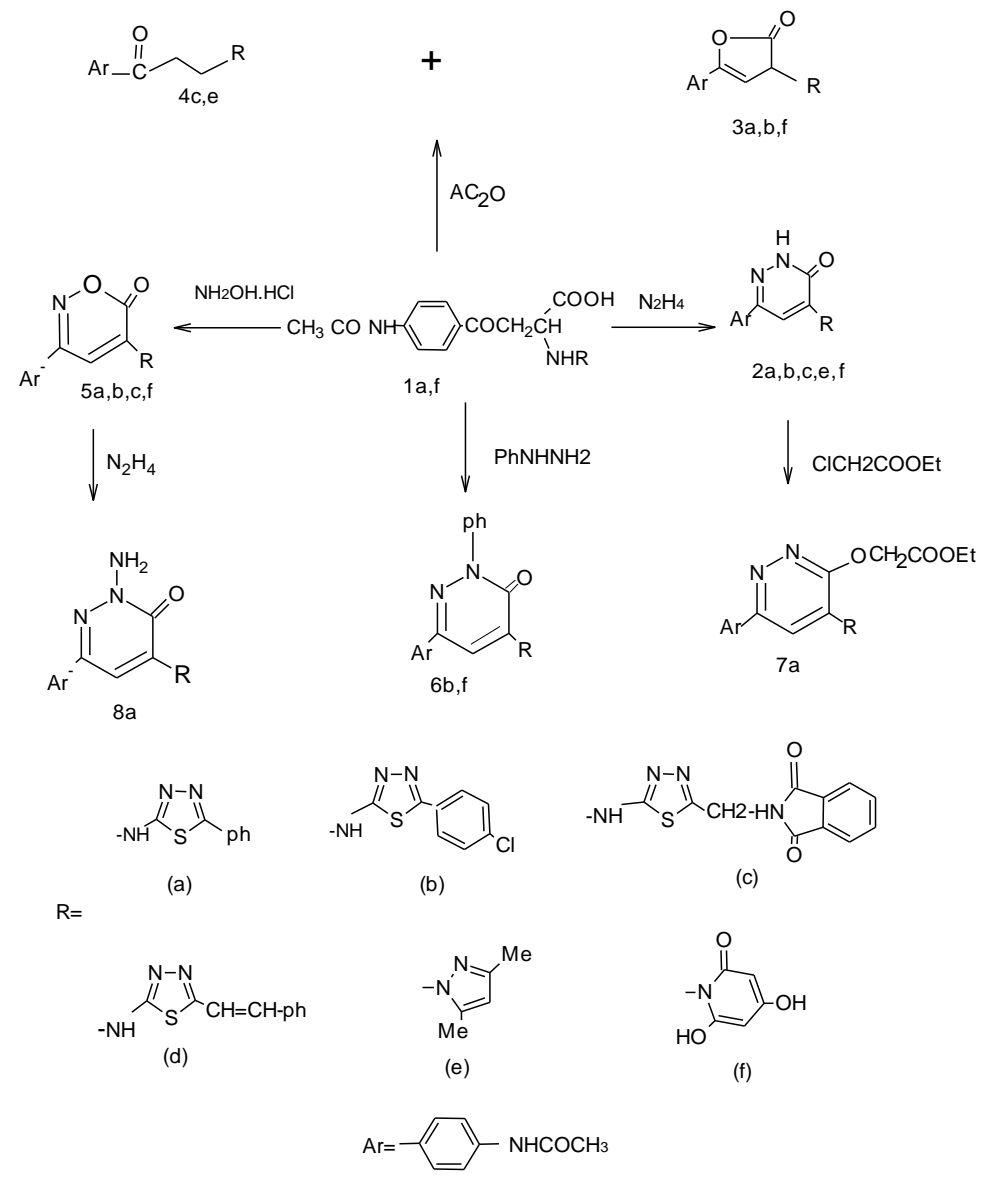

Egypt. J. Chem. 54, No.3 (2011) 


\section{Experimental}

All melting points are uncorrected.Elemental analyses were carried out at the Microanalytical Center, Cairo, Egypt. By Elementar Viro El Microanalysis, IR spectra $(\mathrm{KBr})$ were recorded on infrared spectrometer ST-IR DOMEM Hartman Braun, Model: MBB 157, Canada and H-NMR spectra recorded on a varian $300 \mathrm{MHz}$ (Germany 1999) using TMS as internal standard. The mass spectra were recorded on Shimadzu GCMS-QP-1000 EX mass spectrometer at 70 e.v. Homogeneity of all compounds synthesized was checked by TLC.

\section{General procedure of starting material}

Anhydrous aluminium chloride (200 g) was added portionwise to a stirred solution of Maleic anhydride (100 g) in aromatic hydrocarbon, namely acetanilide $(200 \mathrm{ml})$ in an ice bath. The whole mixture was stirred at room temperature for further $2 \mathrm{hr}$ and refluxed for $3 \mathrm{hr}$ on water bath, then left to stand overnight. The participated solid after addition of ice cold hydrochloric acid $(25 \mathrm{ml})$ was filtered off, dried and the crude product was crystallized form ethanol to give 3-p-acetamidobenzoyl-prop-2-enoic acid.

Formation of 3(p-acetamidobenzoyl)2-(5aryl 2-thiadiazolyl amino)propanoic acids $(1 a-d)$

A solution of 3-(4-acetamidobenzoyl)-prop-2-enoic acid (0.01 mol) and 5aryl-2-amino $1,3,4$-thiadiazole $(0.016 \mathrm{~mol})$ in $30 \mathrm{ml}$ ethanol was refluxed for $3 \mathrm{hr}$. The crude product was washed by petroleum ether (b.p $40-60^{\circ} \mathrm{C}$ ), and then, crystallized from ethanol to give compounds $1 \mathrm{a}, 1 \mathrm{~b}, 1 \mathrm{c}$ or $1 \mathrm{~d}$

Formation of 3-(p-acetamidobenzoyl)2-(5-(3,5 dimethyl)pyrazolo/or barbituro propanoic acid (le,f)

A solution of 3-(4-acetamidoobenzoyl)-prop-2-enoic acid (0.01 mol) (2.55g) and 3,5 dimethyl pyrazole and /or barbituric acid 0.01 mole $(1 \mathrm{~g})$ in $30 \mathrm{ml}$ ethanol in and then refluxed for $5 \mathrm{hr}$. The mixture was washed by light petroleum ether (b.p 40- $60^{\circ} \mathrm{C}$ ), and the solid was crystallized

Formation of 6-acetamidophenyl-4(5-aryl thiazolylamino,3 5 dimethyl pyrazolyl and /or burbituryl)-4,5-dihydro-3(2H)pyridazinones (2)

A solution of $1(0.01 \mathrm{~mol})$ in ethanol $(30 \mathrm{ml})$ was treated with hydrazine hydrate $(0.75 \mathrm{ml})$ and then refluxed for $4 \mathrm{hr}$. The solid that separated after concentration and cooling was recrystallized .

Formation of 5-acetamidophenyl-3-(5-aryl thiazolylamino, and /or burbituryl) furan-2-one $(3 a, b, d, f)$

A solution of $1_{\mathrm{a}, \mathrm{b}, \mathrm{f}}(0.01 \mathrm{~mol})$ and acetic anhydride $(0.01 \mathrm{~mol})(1 \mathrm{ml})$, was heated on water path for $1 \mathrm{hr}$. The product that separated on cooling was recrystallized from suitable solvent to give compounds $3 a, b, d, f$. 
Formation of $\beta$-(5-phthalimido methyl thiazolylamino and/or 3,5 dimethyl pyrazolyl-5-acetamido propiophenone $(4 c, e)$

A solution of $1_{c, e}(0.01 \mathrm{~mol})$ and acetic anhydride $(0.01 \mathrm{~mol})(1 \mathrm{ml})$, was heated under reflux for $1 \mathrm{hr}$. The product that separated on cooling was recrystallized from ethanol to give compound $4 \mathrm{c}, \mathrm{e}$.

Formation of 3-acetamidophenyl-5(5-aryl thiazolylamino,3 5 dimethyl pyrazolyl and /or burbituryl)-4,5,6-trihydro-1,2-oxazine-6-one (5)

A solution of $1_{\mathrm{a}, \mathrm{b}, \mathrm{c}, \mathrm{f}}(0.01 \mathrm{~mole})$ in pyridine $(0.01 \mathrm{~mol})(10 \mathrm{ml})$, was refluxed with hydroxylamine hydrochloride $(0.01 \mathrm{~mol})$ for $3 \mathrm{hr}$. The reaction mixture was left to cool then poured into cold water/HCl. The participated solid was filtered off and recrystallized from the suitable solvents to give compounds 5 .

Formation of 6-acetamidophenyl-4(5-(4-chloro phenyl thiazolyl amino,and /or burbituryl)-1-phenyl-2,3-dihydro-3 $(2 \mathrm{H})$ pyridazinone $(6 \mathrm{~b}, f)$

A solution of $1 \mathrm{~b}, \mathrm{f}(0.01 \mathrm{~mol})$ in ethanol $(30 \mathrm{ml})$ was treated with phenyl hydrazine hydrate $(1.4 \mathrm{ml})$ and then refluxed for $4 \mathrm{hr}$. The solid that separated after concentration and cooling was recrystallized from ethanol to give compounds 6 .

Formation of 6-acetamidophenyl-4(5-phenyl thiazolylamino)-2-ethoxycarbonylmethyl-2,3 - di hydropyridazine-3-one (7)

A solution of $2_{\mathrm{a}}(0.01 \mathrm{~mol})$ and ethylchloroacetate $(0.01 \mathrm{~mol})$ in dry acetone $(20 \mathrm{ml})$, in the presence of potassium carbonate $(3 \mathrm{~g})$ was refluxed for $24 \mathrm{hr}$. The reaction mixture was concentrated, cooled and poured into ice cold water. The participated solid was filtered off, washed, dried and recrystallized from ethanol to give compound 7 .

Formation of 1-amino -6-acetamido phenyl-4(5-phenyl thiazolylamino)- -2,3 - di hydro-3 $(2 \mathrm{H})$ pyridazinone $(8 a)$

A solution of $5 \mathrm{a}(0.01 \mathrm{~mol})$ and hydrazine hydrate $(0.01 \mathrm{~mol})$, was boiled in butanol $(10 \mathrm{ml})$ for $3 \mathrm{hr}$. The reaction mixture was left to cool, then poured into cold water. The participated solid was filtered off and recrystallized from ethanol to give compound $8 \mathrm{a}$. 
TABLE 1. Characterization and physical data for synthesized compound.

\begin{tabular}{|c|c|c|c|c|c|c|c|c|}
\hline \multirow{2}{*}{$\begin{array}{c}\text { Comp. } \\
\text { No }\end{array}$} & \multirow{2}{*}{$\underset{ }{\mathrm{M} . P .}$} & \multirow{2}{*}{$\begin{array}{c}\text { Solvent } \\
\text { of } \\
\text { Cryst. }\end{array}$} & \multirow{2}{*}{ Formula M.W. } & \multicolumn{5}{|c|}{ Analysis \% calcd/found } \\
\hline & & & & $\mathbf{C}$ & $\mathbf{H}$ & $\mathbf{N}$ & Cl & $\mathbf{S}$ \\
\hline $1_{\mathrm{a}}$ & 190 & Ethanol & $\mathrm{C}_{20} \mathrm{H}_{18} \mathrm{~N}_{4} \mathrm{O}_{4} \mathrm{~S}(410)$ & $\begin{array}{l}58.5 \\
58.4\end{array}$ & $\begin{array}{c}4.4 \\
4.25\end{array}$ & $\begin{array}{l}13.7 \\
13.9\end{array}$ & - & $\begin{array}{l}7.8 \\
7.9\end{array}$ \\
\hline $1_{\mathrm{b}}$ & 250 & Ethanol & $\mathrm{C}_{20} \mathrm{H}_{17} \mathrm{~N}_{4} \mathrm{O}_{4} \mathrm{SCl}(445)$ & $\begin{array}{l}53.9 \\
53.7\end{array}$ & $\begin{array}{l}3.8 \\
3.7\end{array}$ & $\begin{array}{l}12.5 \\
12.7\end{array}$ & $\begin{array}{l}8.00 \\
8.23 \\
\end{array}$ & $\begin{array}{l}7.4 \\
7.4 \\
\end{array}$ \\
\hline $1_{\mathrm{C}}$ & 210 & Ethanol & $\mathrm{C}_{23} \mathrm{H}_{19} \mathrm{~N}_{5} \mathrm{O}_{6} \mathrm{~S}(493)$ & $\begin{array}{l}55.9 \\
55.6\end{array}$ & $\begin{array}{l}3.9 \\
3.7\end{array}$ & $\begin{array}{l}14.2 \\
14.3\end{array}$ & - & $\begin{array}{l}6.6 \\
6.4\end{array}$ \\
\hline $1_{\mathrm{d}}$ & 255 & Ethanol & $\mathrm{C}_{22} \mathrm{H}_{20} \mathrm{~N}_{4} \mathrm{O}_{4} \mathrm{~S}(436)$ & $\begin{array}{l}60.5 \\
60.4\end{array}$ & $\begin{array}{l}4.6 \\
4.5\end{array}$ & $\begin{array}{l}12.8 \\
12.7\end{array}$ & - & $\begin{array}{l}7.3 \\
7.3\end{array}$ \\
\hline $1_{\mathrm{e}}$ & 254 & Ethanol & $\mathrm{C}_{17} \mathrm{H}_{19} \mathrm{~N}_{3} \mathrm{O}_{4}(329)$ & $\begin{array}{l}62.0 \\
62.2\end{array}$ & $\begin{array}{l}5.9 \\
5.7\end{array}$ & $\begin{array}{l}12.9 \\
12.8\end{array}$ & - & - \\
\hline $1_{\mathrm{f}}$ & 300 & Dioxan & $\mathrm{C}_{16} \mathrm{H}_{15} \mathrm{~N}_{3} \mathrm{O}_{7}(360)$ & $\begin{array}{l}53.3 \\
53.2\end{array}$ & $\begin{array}{l}4.3 \\
4.2 \\
\end{array}$ & $\begin{array}{l}11.7 \\
11.7\end{array}$ & - & - \\
\hline $2 \mathrm{a}$ & 190 & Ethanol & $\mathrm{C}_{20} \mathrm{H}_{18} \mathrm{~N}_{4} \mathrm{O}_{4} \mathrm{~S}(410)$ & $\begin{array}{l}58.5 \\
58.4\end{array}$ & $\begin{array}{l}4.5 \\
4.5\end{array}$ & $\begin{array}{l}13.7 \\
13.6\end{array}$ & - & $\begin{array}{l}7.8 \\
7.7\end{array}$ \\
\hline $2 \mathrm{~b}$ & 300 & Ethanol & $\mathrm{C}_{20} \mathrm{H}_{17} \mathrm{~N}_{6} \mathrm{O}_{2} \mathrm{SCl}(441)$ & $\begin{array}{l}54.4 \\
54.4\end{array}$ & $\begin{array}{l}3.9 \\
3.8\end{array}$ & $\begin{array}{l}19.0 \\
18.8\end{array}$ & $\begin{array}{l}8.00 \\
7.87\end{array}$ & $\begin{array}{l}7.3 \\
7.5\end{array}$ \\
\hline $2 c_{c}$ & 270 & Ethanol & $\mathrm{C}_{23} \mathrm{H}_{19} \mathrm{~N}_{7} \mathrm{O}_{4} \mathrm{~S}(489)$ & $\begin{array}{l}56.4 \\
56.2\end{array}$ & $\begin{array}{l}3.9 \\
3.9\end{array}$ & $\begin{array}{l}20.0 \\
19.7\end{array}$ & - & $\begin{array}{l}6.5 \\
6.3\end{array}$ \\
\hline $2 e_{\mathrm{e}}$ & 300 & Ethanol & $\mathrm{C}_{17} \mathrm{H}_{19} \mathrm{~N}_{5} \mathrm{O}_{2}(325)$ & $\begin{array}{l}62.8 \\
62.5\end{array}$ & $\begin{array}{l}5.8 \\
5.6\end{array}$ & $\begin{array}{l}21.5 \\
21.3\end{array}$ & - & - \\
\hline $2 \mathrm{f}$ & 225 & DMF & $\mathrm{C}_{16} \mathrm{H}_{15} \mathrm{~N}_{5} \mathrm{O}_{5}(357)$ & $\begin{array}{l}53.9 \\
53.6\end{array}$ & $\begin{array}{l}4.2 \\
4.5 \\
\end{array}$ & $\begin{array}{l}19.6 \\
19.5\end{array}$ & - & - \\
\hline $3 \mathrm{a}$ & 210 & Dioxan & $\mathrm{C}_{20} \mathrm{H}_{17} \mathrm{~N}_{4} \mathrm{O}_{3} \mathrm{~S}(391)$ & $\begin{array}{l}61.5 \\
61.4\end{array}$ & $\begin{array}{l}4.3 \\
4.2\end{array}$ & $\begin{array}{l}14.3 \\
14.3\end{array}$ & - & $\begin{array}{l}8.3 \\
8.2\end{array}$ \\
\hline $3 \mathrm{~b}$ & 300 & Ethanol & $\mathrm{C}_{20} \mathrm{H}_{15} \mathrm{~N}_{4} \mathrm{O}_{3} \mathrm{Cl}(427)$ & $\begin{array}{l}56.2 \\
56.1\end{array}$ & $\begin{array}{l}3.5 \\
3.7\end{array}$ & $\begin{array}{l}13.1 \\
13.4\end{array}$ & $\begin{array}{l}8.31 \\
7.97\end{array}$ & - \\
\hline $3_{\mathrm{f}}$ & 302 & Ethanol & $\mathrm{C}_{16} \mathrm{H}_{13} \mathrm{~N}_{3} \mathrm{O}_{6}(346)$ & $\begin{array}{l}55.7 \\
55.4\end{array}$ & $\begin{array}{l}3.8 \\
3.7\end{array}$ & $\begin{array}{l}12.0 \\
11.9\end{array}$ & - & - \\
\hline $4_{c}$ & 270 & Dioxan & $\mathrm{C}_{22} \mathrm{H}_{20} \mathrm{~N}_{5} \mathrm{O}_{4} \mathrm{~S}(450)$ & $\begin{array}{l}58.7 \\
58.4\end{array}$ & $\begin{array}{l}4.4 \\
4.2\end{array}$ & $\begin{array}{l}15.5 \\
15.2\end{array}$ & - & $\begin{array}{l}7.1 \\
6.8\end{array}$ \\
\hline $4 \mathrm{e}_{\mathrm{e}}$ & 280 & Ethanol & $\mathrm{C}_{16} \mathrm{H}_{19} \mathrm{~N}_{3} \mathrm{O}_{2}(285)$ & $\begin{array}{l}67.3 \\
67.1\end{array}$ & $\begin{array}{l}6.7 \\
6.4\end{array}$ & $\begin{array}{l}14.7 \\
14.3\end{array}$ & - & - \\
\hline $5_{\mathrm{a}}$ & 255 & Ethanol & $\mathrm{C}_{20} \mathrm{H}_{16} \mathrm{~N}_{5} \mathrm{O}_{3} \mathrm{~S}(406)$ & $\begin{array}{l}59.1 \\
59.5\end{array}$ & $\begin{array}{l}3.9 \\
3.5\end{array}$ & $\begin{array}{l}17.2 \\
16.9\end{array}$ & - & $\begin{array}{l}7.9 \\
7.5 \\
\end{array}$ \\
\hline $5 \mathrm{~b}$ & 140 & Ethanol & $\mathrm{C}_{20} \mathrm{H}_{15} \mathrm{~N}_{5} \mathrm{O}_{3} \mathrm{SCl}(441)$ & $\begin{array}{l}59.4 \\
59.7\end{array}$ & $\begin{array}{l}3.4 \\
3.6\end{array}$ & $\begin{array}{l}15.9 \\
15.5\end{array}$ & $\begin{array}{l}8.05 \\
7.78\end{array}$ & $\begin{array}{l}7.3 \\
7.5\end{array}$ \\
\hline $5_{\mathrm{c}}$ & 282 & Dioxan & $\mathrm{C}_{23} \mathrm{H}_{18} \mathrm{~N}_{6} \mathrm{O}_{5} \mathrm{~S}(490)$ & $\begin{array}{l}56.3 \\
56.3\end{array}$ & $\begin{array}{l}3.6 \\
3.4\end{array}$ & $\begin{array}{l}17.1 \\
16.8\end{array}$ & - & $\begin{array}{l}6.5 \\
6.4\end{array}$ \\
\hline $5_{\mathrm{f}}$ & 305 & Ethanol & $\mathrm{C}_{16} \mathrm{H}_{13} \mathrm{~N}_{4} \mathrm{O}_{6}(357)$ & $\begin{array}{l}53.9 \\
53.4\end{array}$ & $\begin{array}{l}3.6 \\
3.5\end{array}$ & $\begin{array}{l}15.8 \\
15.4\end{array}$ & - & - \\
\hline $6 \mathrm{~b}$ & 162 & Ethanol & $\mathrm{C}_{26} \mathrm{H}_{20} \mathrm{~N}_{6} \mathrm{O}_{2} \mathrm{~S}(516)$ & $\begin{array}{l}60.5 \\
60.2\end{array}$ & $\begin{array}{l}3.9 \\
4.2 \\
\end{array}$ & $\begin{array}{l}16.4 \\
16.7\end{array}$ & - & $\begin{array}{l}6.2 \\
6.3 \\
\end{array}$ \\
\hline $6_{\mathrm{f}}$ & 220 & Ethanol & $\mathrm{C}_{22} \mathrm{H}_{19} \mathrm{~N}_{5} \mathrm{O} 5(433)$ & $\begin{array}{l}61.0 \\
61.5\end{array}$ & $\begin{array}{l}4.5 \\
4.8\end{array}$ & $\begin{array}{l}16.3 \\
16.5\end{array}$ & - & - \\
\hline $7 \mathrm{a}$ & 250 & Ethanol & $\mathrm{C}_{24} \mathrm{H}_{24} \mathrm{~N}_{6} \mathrm{O}_{4} \mathrm{~S}(392)$ & $\begin{array}{l}58.5 \\
58.8\end{array}$ & $\begin{array}{l}4.9 \\
4.3\end{array}$ & $\begin{array}{l}17.1 \\
17.4\end{array}$ & - & $\begin{array}{l}6.5 \\
6.2\end{array}$ \\
\hline $8 \mathrm{a}$ & 302 & Ethanol & $\mathrm{C}_{20} \mathrm{H}_{19} \mathrm{~N}_{7} \mathrm{O}_{2} \mathrm{~S}(421)$ & $\begin{array}{l}57.0 \\
57.4\end{array}$ & $\begin{array}{l}4.5 \\
4.3\end{array}$ & $\begin{array}{l}23.5 \\
23.1\end{array}$ & - & $\begin{array}{l}7.6 \\
7.4\end{array}$ \\
\hline
\end{tabular}




\section{Biological Screening}

The antimicrobial screening of all the synthesized compounds was done using the agar diffusion assay. This screening was performed against the Grampositive bacteria, Gram-negative bacteria, Staphylococcus aureus Atcc 06538, Escherechia coli Atcc 10536, pathogenic fungi Candida albicans Atcc 1023 and Aspergills flavus. A moderate activity was observed with compounds which proved to possess marked activity against $E$. coli, S. aureus and $C$. albicans. The strong activity was observed with compound $6 \mathrm{c}$. The inhibitory concentration was determined for each of the active compounds along with Tetracycline and Amphotericin as positive control. Activity was detected for the synthesized compounds except 1e, 3d and 4c toward Aspergillus flavus. Results are shown in the Table 2 .

TABLE 2. Antibacterial and antifungal activities of some selected compounds.

\begin{tabular}{|c|c|c|c|c|c|}
\hline \multicolumn{2}{|c|}{ Sample } & $\begin{array}{l}\text { Escherichia coli } \\
\left(\mathbf{G}^{-}\right)\end{array}$ & $\begin{array}{c}\text { Staphylococcus } \\
\text { aureus } \\
\left(\mathbf{G}^{+}\right)\end{array}$ & $\begin{array}{l}\text { Aspergillus } \\
\text { flavus } \\
\text { Fungus }\end{array}$ & $\begin{array}{l}\text { Candida } \\
\text { albicans } \\
\text { Fungus }\end{array}$ \\
\hline \multicolumn{2}{|c|}{ Control : DMSO } & 0.0 & 0.0 & 0.0 & 0.0 \\
\hline \multirow[t]{2}{*}{$\begin{array}{l}\text { Stan- } \\
\text { dard }\end{array}$} & $\begin{array}{l}\text { Tetracy- } \\
\text { cline } \\
\text { Anti- } \\
\text { bacterial }\end{array}$ & 32 & 30 & - & - \\
\hline & $\begin{array}{l}\text { Ampho- } \\
\text { tericin } \\
\text { B } \\
\text { Antifungal }\end{array}$ & - & - & 16 & 18 \\
\hline \multicolumn{2}{|r|}{$1_{\mathrm{a}}$} & 14 & 14 & 12 & 10 \\
\hline \multicolumn{2}{|r|}{$1_{\mathrm{b}}$} & 14 & 14 & 14 & 12 \\
\hline \multicolumn{2}{|r|}{$1_{\mathrm{c}}$} & 15 & 14 & 14 & 12 \\
\hline \multicolumn{2}{|r|}{$1_{\mathrm{d}}$} & 14 & 13 & 14 & 11 \\
\hline \multicolumn{2}{|r|}{$1_{\mathrm{e}}$} & 14 & 13 & 0.0 & 0.0 \\
\hline \multicolumn{2}{|r|}{$3 \mathrm{~b}$} & 13 & 13 & 13 & 10 \\
\hline \multicolumn{2}{|r|}{$3_{\mathrm{d}}$} & 14 & 15 & 0.0 & 10 \\
\hline \multicolumn{2}{|r|}{$4_{c}$} & 13 & 14 & 0.0 & 0.0 \\
\hline \multicolumn{2}{|r|}{$5 \mathrm{~b}$} & 13 & 12 & 13 & 12 \\
\hline \multicolumn{2}{|r|}{$6_{c}$} & 16 & 16 & 13 & 12 \\
\hline
\end{tabular}




\begin{tabular}{|c|c|c|}
\hline $\begin{array}{l}\text { Comp. } \\
\text { No }\end{array}$ & IR & NMR.(DMSO) \\
\hline $1_{\mathrm{a}}$ & $\begin{array}{l}\text { vOH3410 vNH } 3297-3031,3050- \\
2892(\mathrm{C}-\mathrm{H} 1710) \text { and }\left(1680 \mathrm{~cm}^{-1}\right) \\
\text { acid and ketone groups }\end{array}$ & $\begin{array}{l}2.5\left(\mathrm{~s}, 3 \mathrm{H}, \mathrm{CH}_{3} \mathrm{CO}\right), 3.4\left(\mathrm{oct}, \mathrm{CH}_{2-} \mathrm{C}=\mathrm{O} \mathrm{J}=\right. \\
15.2, \mathrm{~J}=7.7)(\text { diastereotopic protons }) \\
4.2(\mathrm{dd}, \mathrm{CH}-\mathrm{COOH}, \text { methin proton } \mathrm{J}=7.7) 6.7 \\
(\mathrm{~s}, \mathrm{NH}), 7.4-7.8(\mathrm{~m}, 9 \mathrm{H}, \mathrm{ArH}), 8.2 \\
(\mathrm{~s}, 1 \mathrm{H}, \mathrm{COOH}), 8.6(\mathrm{~s}, 1 \mathrm{H}, \mathrm{C}=\mathrm{O}-\mathrm{NH})\end{array}$ \\
\hline $1_{\mathrm{b}}$ & $\begin{array}{l}\text { vNH or } \mathrm{OH} 3150-3320,3050-2905 \\
(\mathrm{C}-\mathrm{H}) 1710) \text { and }\left(1680 \mathrm{~cm}^{-1}\right) \text { acid } \\
\text { and ketone groups }\end{array}$ & $\begin{array}{l}2.5\left(\mathrm{~s}, 3 \mathrm{H}, \mathrm{CH}_{3} \mathrm{CO}\right), 3.4\left(\text { oct }, \mathrm{CH}_{2-} \mathrm{C}=\mathrm{O} \mathrm{J}=15.2\right. \\
\mathrm{J}=7.7)(\text { diastereotopic proton }) 4.2(\mathrm{dd}, \mathrm{CH}- \\
\mathrm{COOH}, \text { methin proton } \mathrm{J}=7.7) 6.7(\mathrm{~s}, \mathrm{NH}), 7.6- \\
8.1(\mathrm{~m}, 8 \mathrm{H}, \mathrm{ArH}), 8.2(\mathrm{~s}, 1 \mathrm{H}, \mathrm{COOH}), 8.6 \\
(\mathrm{~s}, 1 \mathrm{H}, \mathrm{C}=\mathrm{O}-\mathrm{NH})\end{array}$ \\
\hline $1_{\mathrm{c}}$ & $\begin{array}{l}\text { vNHor OH } 3327-3031,3055- \\
2912\left(\mathrm{C}-\mathrm{H}\left(1770-1712 \mathrm{~cm}^{-1}\right)\right. \\
\text { vCO phthalimido moiety, }(1710) \\
\text { and }\left(1680 \mathrm{~cm}^{-1}\right) \text { corresponding to } \\
\text { acid and ketone groups }\end{array}$ & $\begin{array}{l}2.45\left(\mathrm{~s}, 3 \mathrm{H}, \mathrm{CH}_{3} \mathrm{CO}\right), 3.4\left(\text { oct }, \mathrm{CH}_{2} \mathrm{C}=\mathrm{O}\right. \\
\mathrm{J}=15.2, \mathrm{~J}=7.7)(\text { diastereotopic protons }) \\
4.1(\mathrm{dd}, \mathrm{CH}-\mathrm{COOH}, \text { methinproton } \mathrm{J}=7.7) 5.2 \\
(\mathrm{~s}, 2 \mathrm{H}, \mathrm{CH} 2) 6.2(\mathrm{~s}, \mathrm{NH}), 7.4-7.8(\mathrm{~m}, 8 \mathrm{H}, \mathrm{ArH}), \\
8.2(\mathrm{~s}, 1 \mathrm{H}, \mathrm{COOH}), 8.6(\mathrm{~s}, 1 \mathrm{H}, \mathrm{C}=\mathrm{O}-\mathrm{NH})\end{array}$ \\
\hline $1_{\mathrm{d}}$ & $\begin{array}{l}\text { vNHor OH } 3031-3297,3022-2912 \\
\left(\mathrm{C}-\mathrm{H}(1710) \text { and }\left(1680 \mathrm{~cm}^{-1}\right)\right. \\
\text { corresponding to acid and ketone } \\
\text { group respectively. }\end{array}$ & $\begin{array}{l}\left.\text { 2.5(s,3H, } \mathrm{CH}_{3} \mathrm{CO}\right), 3.4\left(\text { oct, } \mathrm{CH}_{2-} \mathrm{C}=\mathrm{O} \mathrm{J}=7.7\right) \\
(\mathrm{J}=15.2, \text { diastereotopic protons }) 4.2(\mathrm{dd}, \mathrm{CH}- \\
\mathrm{COOH}, \text { methinproton } \mathrm{J}=7.7) 5.3(\mathrm{~d}, 1 \mathrm{H}, \mathrm{Ph}- \\
\mathrm{CHa}=), 5.6(\mathrm{~d},=\mathrm{CHb}) 6.5(\mathrm{~s}, \mathrm{NH}), 7.4-7.8 \\
(\mathrm{~m}, 9 \mathrm{H}, \mathrm{ArH}), 8.2(\mathrm{~s}, 1 \mathrm{H}, \mathrm{COOH}), 8.6 \\
(\mathrm{~s}, 1 \mathrm{H}, \mathrm{C}=\mathrm{O}-\mathrm{NH})\end{array}$ \\
\hline $1_{\mathrm{e}}$ & $\begin{array}{l}\text { vNHor OH } 3031-3297(\mathrm{NH}), 3029- \\
2892 \mathrm{C}-\mathrm{H}(1710) \text { and }\left(1680 \mathrm{~cm}^{-1}\right) \\
\text { corresponding to acid and ketone } \\
\text { group respectively. }\end{array}$ & 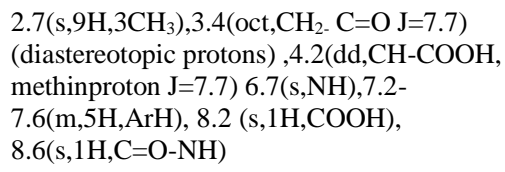 \\
\hline $1_{\mathrm{f}}$ & $\begin{array}{l}\text { vNHor OH } 3450-3130,3050-2900 \\
(\mathrm{C}-\mathrm{H} \text { ( } 1710) \text { and }\left(1680 \mathrm{~cm}^{-1}\right) \\
\text { corresponding to acid and ketone } \\
\text { group respectively and }(1615) \\
\text { corresponding to } \\
\text { barbiturate moiety. }\end{array}$ & $\begin{array}{l}\text { 2.5 }\left(\mathrm{s}, 3 \mathrm{H}, \mathrm{CH}_{3} \mathrm{CO}\right), 3.4\left(\text { oct }, \mathrm{CH}_{2-} \mathrm{C}=\mathrm{O} \mathrm{J}=7.7\right) \\
\text { (diastereotopic protons } \mathrm{J}=15.2) \\
\text { 4.2(dd,CH-COOH, methinproton } \mathrm{J}=7.7) \\
6.7(\mathrm{~s}, \mathrm{NH}), 7.1-7.5(\mathrm{~m}, 6 \mathrm{H}, \mathrm{ArH}), \\
8.2(\mathrm{~s}, 1 \mathrm{H}, \mathrm{COOH}), 8.6(\mathrm{bs}, 2 \mathrm{H}, \mathrm{C}=\mathrm{O}-\mathrm{NH}) \\
\text { Acetamido and barbiturate moieties }\end{array}$ \\
\hline $2 \mathrm{a}$ & $\begin{array}{l}\text { vNH } 3297-3125,3055-2900 \mathrm{C}-\mathrm{H} \\
\left(1640 \mathrm{~cm}^{-1}\right) \text { for } 2 v \mathrm{CO} \text { of amide } \\
\text { group }\end{array}$ & $\begin{array}{l}2.5(\mathrm{~s}, 3 \mathrm{H}, \mathrm{CH} 3), 6.7(\mathrm{~s}, \mathrm{NH}) 7.4-7.8 \\
(\mathrm{~m}, 9 \mathrm{H}, \mathrm{ArH}) 7.2(\mathrm{~s}, 1 \mathrm{H}, \text { proton of } \\
\text { pyridazinone, } 10.2(\mathrm{~s}, 2 \mathrm{H}, \mathrm{NH} \text { acidic } \\
\text { protons), }\end{array}$ \\
\hline $2 \mathrm{~b}$ & $\begin{array}{l}\text { vNH } 3270-3150,3047-2882 \mathrm{C}-\mathrm{H} \\
\left(1640 \mathrm{~cm}^{-1}\right) \text { for } 2 v \mathrm{CO} \text { of amide } \\
\text { group }\end{array}$ & $\begin{array}{l}2.5(\mathrm{~s}, 3 \mathrm{H}, \mathrm{CH} 3), 6.7(\mathrm{~s}, \mathrm{NH}) 7.4-8.05 \\
(\mathrm{~m}, 8 \mathrm{H}, \mathrm{ArH}) 7.2(\mathrm{~s}, 1 \mathrm{H}, \text { proton of } \\
\text { pyridazinone, } 10.2(\mathrm{~s}, 2 \mathrm{H}, \mathrm{NH} \text { acidic } \\
\text { protons })\end{array}$ \\
\hline $2{ }_{c}$ & $\begin{array}{l}\text { vNH } 3300-3080,3022-2912 \mathrm{C}-\mathrm{H} \\
\left(1640 \mathrm{~cm}^{-1}\right) \text { for } 2 v \mathrm{CO} \text { of amide } \\
\text { group }(1772-1712) v \mathrm{CO} \text { in } \\
\text { phthalimido moiety }\end{array}$ & $\begin{array}{l}2.5(\mathrm{~s}, 3 \mathrm{H}, \mathrm{CH} 3), 6.7(\mathrm{~s}, \mathrm{NH}), 6.8-7.9 \\
\text { (m,8H,ArH) 5.2 (s, 2H, CH2), } 7.2 \\
\text { (s,1H,proton pyridazinone), } \\
10.2 \text { (s,2H,NH acidic protons), }\end{array}$ \\
\hline
\end{tabular}

Egypt. J. Chem. 54, No.3 (2011) 


\begin{tabular}{|c|c|c|}
\hline $\begin{array}{l}\text { Comp. } \\
\text { No }\end{array}$ & IR & NMR. (DMSO) \\
\hline $2 e_{e}$ & $\begin{array}{l}\text { vNH } 3297-3031,3060-2905 \mathrm{C}-\mathrm{H} \\
\left(1640 \mathrm{~cm}^{-1}\right) \text { for } 2 \mathrm{v} \mathrm{CO} \text { of amide } \\
\text { group of pyridazinone and } \\
\text { acetamido moietes }\end{array}$ & $\begin{array}{l}2.6(\mathrm{~s}, 9 \mathrm{H}, 3 \mathrm{CH} 3), 6.8-7.2(\mathrm{~m}, 4 \mathrm{H}, \mathrm{ArH}) \\
7.5-7.6(\mathrm{~s}, 2 \mathrm{H}, \text { protons of pyrazole and } \\
\text { pyridazinone }), 10.2(\mathrm{~s}, 2 \mathrm{H}, \mathrm{NH} \text { acidic } \\
\text { protons })\end{array}$ \\
\hline $2_{\mathrm{f}}$ & $\begin{array}{l}\mathrm{vNH} 3297-3120,3050-2912 \mathrm{C}-\mathrm{H} \\
\left(1640 \mathrm{~cm}^{-1}\right) \text { for } \mathrm{v} \text { CO of amide of } \\
\text { pyrimidinonyl and pyridazinone } \\
\text { moieties }\end{array}$ & $\begin{array}{l}2.3(\mathrm{~s}, 3 \mathrm{H}, \mathrm{CH} 3), 6.8-7.3(\mathrm{~m}, 4 \mathrm{H}, \mathrm{ArH}) 7.5- \\
7.8(\mathrm{~m}, 3 \mathrm{H}, \text { protons of pyridazinone and } \\
\text { pyrimidine moieties), } 10.2(\mathrm{~s}, 2 \mathrm{H}, \mathrm{NH} \text { acidic } \\
\text { protons }), 11.2(\mathrm{~s}, 2 \mathrm{H}, \mathrm{OH})\end{array}$ \\
\hline $3 \mathrm{a}$ & $\begin{array}{l}\text { vNH } 3297-3100,3055-2890(\mathrm{C}-\mathrm{H}) \\
1767 \mathrm{~cm}^{-1} \text { attributable to v CO } \\
\text { lactonic and acetamido gp at } 1693 \\
\mathrm{~cm}^{-1}\end{array}$ & $\begin{array}{l}\delta 2,1\left(\mathrm{~s} 3 \mathrm{H}, \mathrm{CH}_{3} \mathrm{CO}\right), 4(\mathrm{dd} 1 \mathrm{H},-\mathrm{CH}-\mathrm{HH} \\
\mathrm{J}=8.5), 6.7(\mathrm{bs}, \mathrm{NH}), 7.5-7.9(\mathrm{~m}, 9 \mathrm{H} \text { of } \mathrm{Ar}) \\
6.9(\mathrm{~d}, 1 \mathrm{H}, \mathrm{CH} \text { furanone moiety J=8.5), } 12.7 \\
(\mathrm{s}, 1 \mathrm{H}-\mathrm{C}=\mathrm{O}-\mathrm{N} \underline{\mathrm{H}}) \text { acidic protons are } \\
\text { exchangeable in } \mathrm{D}_{2} \mathrm{O},\end{array}$ \\
\hline $3 \mathrm{~b}$ & $\begin{array}{l}\text { vNH } 3297-3031,3022-2900(\mathrm{C}-\mathrm{H} \\
\left(1755 \mathrm{~cm}^{-1}\right) \text { attributable to v CO } \\
\text { (lactonic ) and acetamido gp at } \\
1693 \mathrm{~cm}^{-1}\end{array}$ & $\begin{array}{l}\delta 2,1\left(\mathrm{~s} 3 \mathrm{H}, \mathrm{CH}_{3} \mathrm{CO}\right), 4,(\mathrm{dd} 1 \mathrm{H},-\mathrm{CH}-\mathrm{NH} \\
\mathrm{J}=7.8), 6.3(\mathrm{~s}, \mathrm{NH}) 7.0-7.9(\mathrm{~m}, 8 \mathrm{H} \text { of } \mathrm{Ar}), 6.7 \\
(\mathrm{~d}, 1 \mathrm{H}, \mathrm{CH} \text { furanone moiety J=7.8),12.2 } \\
(\mathrm{s}, 1 \mathrm{H}-\mathrm{C}=\mathrm{O}-\mathrm{N} \underline{\mathrm{H}}) \text { acidic protons }\end{array}$ \\
\hline $3_{\mathrm{f}}$ & $\begin{array}{l}\text { vNH } 3297-3150,3050-2912(\mathrm{C}-\mathrm{H} \\
\left(1760 \mathrm{~cm}^{-1}\right) \text { attributable to v } \mathrm{CO} \\
\text { (lactonic ) and acetamido gp at } 1693 \\
\mathrm{~cm}^{-1}\end{array}$ & $\begin{array}{l}\delta 2,1\left(\mathrm{~s} 3 \mathrm{H}, \mathrm{CH}_{3} \mathrm{CO}\right), 4.3(\mathrm{dd} 1 \mathrm{H},-\mathrm{CH}- \\
\mathrm{NH}), 6.7(\mathrm{~s}, \mathrm{NH}), 7.5-7.9(\mathrm{~m}, 6 \mathrm{H} \text { of } \mathrm{Ar}), \\
(\mathrm{d}, 1 \mathrm{H}, \mathrm{CH} \text { furanone moiety) }, 12.2 \text { (s,1H - } \\
\mathrm{C}=\mathrm{O}-\mathrm{NH}), 13.1(\mathrm{~s}, 2 \mathrm{H}, \mathrm{OH}) \text { acidic protons } \\
\text { exchangeable } \mathrm{D}_{2} \mathrm{O},\end{array}$ \\
\hline $4_{c}$ & $\begin{array}{l}\text { vNH } 3300-3100,3050-2910 \mathrm{CH} \\
\left(1706 \mathrm{~cm}^{-1}\right) \text { corresponding to } v \mathrm{CO} \\
\text { of ketones }\end{array}$ & $\begin{array}{l}2.5\left(\mathrm{~s}, 3 \mathrm{H}, \mathrm{CH}_{3} \mathrm{CO}\right), 3.4\left(\mathrm{t}, \mathrm{CH}_{2} \mathrm{C}=\mathrm{O} \mathrm{J}=7.7\right) 4.1 \\
\left(\mathrm{t}, \mathrm{CH}_{2} \mathrm{~N} \mathrm{~J}=7.7\right), 6.7(\mathrm{bs}, \mathrm{NH}), 7.0-7.8 \\
(\mathrm{~m}, 8 \mathrm{H}, \mathrm{ArH}), 8.6(\mathrm{~s}, 1 \mathrm{H}, \mathrm{C}=\mathrm{O}-\mathrm{NH})\end{array}$ \\
\hline $4_{\mathrm{e}}$ & $\begin{array}{l}\text { vNH } 3297-30150,3020-2912(\mathrm{C}- \\
\mathrm{H} 3031-3297,3022-2912(\mathrm{C}-\mathrm{H} \\
\left(1706 \mathrm{~cm}^{-1}\right) \text { corresponding to } v \\
\text { CO of ketones }\end{array}$ & $\begin{array}{l}2.4\left(\mathrm{~s}, 9 \mathrm{H}, 3 \mathrm{CH}_{3}\right), 3.4\left(\mathrm{t}, \mathrm{CH}_{2} \mathrm{C}=\mathrm{OJ}=7.7\right)(4.1 \\
\left(\mathrm{t}, \mathrm{CH}_{2} \mathrm{~N} \mathrm{~J}=7.7\right), 6.7(\mathrm{bs}, \mathrm{NH}), 7.4-7.8(\mathrm{~m}, 5 \mathrm{H}, \\
\mathrm{ArH} \text { and pyrazole moiety), 8.6 (s,1H,C=O- } \\
\mathrm{NH})\end{array}$ \\
\hline $5 \mathrm{a}$ & $\begin{array}{l}\text { vNH } 3297-3031,3052-2912(\mathrm{C}- \\
\mathrm{H} 1712-1703 \mathrm{~cm}^{-1} \text { corresponding v } \\
\text { CO of azalactone }\end{array}$ & $\begin{array}{l}2.5\left(\mathrm{~s}, 3 \mathrm{H}, \mathrm{CH}_{3} \mathrm{CO}\right), 6.5(\mathrm{~s}, \mathrm{NH}), 7.1-8.2 \\
(\mathrm{~m}, 10 \mathrm{H}, \mathrm{ArH} \text { and oxazine proton }), 8.6 \\
(\mathrm{~s}, 1 \mathrm{H}, \mathrm{C}=\mathrm{O}-\mathrm{NH})\end{array}$ \\
\hline $5 \mathrm{~b}$ & $\begin{array}{l}\text { vNH } 3240-3111,3022-2912(\mathrm{C}- \\
\mathrm{H} 1712-1703 \mathrm{~cm}^{-1} \text { correspondin v } \\
\text { CO of azalactone }\end{array}$ & $\begin{array}{l}2.5\left(\mathrm{~s}, 3 \mathrm{H}, \mathrm{CH}_{3} \mathrm{CO}\right), 6.7(\mathrm{~s}, \mathrm{NH}), 7.1-7.9(\mathrm{~m}, \\
9 \mathrm{H}, \mathrm{ArH} \text { and oxazine proton }), 8.6(\mathrm{~s}, 1 \mathrm{H}, \\
\mathrm{C}=\mathrm{O}-\mathrm{NH})\end{array}$ \\
\hline $5 \mathrm{c}$ & $\begin{array}{l}\text { vNH } 3270-3030,3060-2912(\mathrm{C}-\mathrm{H}) \text {, } \\
\text { v CO of azalactone } 1712-1703 \mathrm{~cm}^{-1} \\
\text { v CO at1772-1712 for phthalimido } \\
\text { moieties }\end{array}$ & $\begin{array}{l}2.5\left(\mathrm{~s}, 3 \mathrm{H}, \mathrm{CH}_{3} \mathrm{CO}\right), 5.2(\mathrm{~s}, 2 \mathrm{H}, \mathrm{CH} 2), 6.7 \\
\text { (s,NH), 7.1-7.9 (m,9H,ArH and oxazine } \\
\text { proton), 8.6(s,1H,C=O-NH) }\end{array}$ \\
\hline $5_{\mathrm{f}}$ & $\begin{array}{l}\text { vNH 3290-3100, 3050-2912(C-H) } \\
1712,1703,1683 \mathrm{~cm}^{-1} \text { v CO of } \\
\text { azalactone, v CO barbituro and } \\
\text { pyrimidinone moieties }\end{array}$ & $\begin{array}{l}2.5\left(\mathrm{~s}, 3 \mathrm{H}, \mathrm{CH}_{3} \mathrm{CO}\right), 6.7(\mathrm{~s}, \mathrm{NH}), 6.9-8.3(\mathrm{~m}, 8 \mathrm{H}, \\
\text { ArH Pyrimidine and oxazine protons }), 9.9 \\
(\mathrm{bs}, 1 \mathrm{H}, \mathrm{OH} \text { acidic proton and } 12.4(\mathrm{~s}, 1 \mathrm{H}, \mathrm{C} \\
\left.=\mathrm{ONH} \text { are exchangeable in } \mathrm{D}_{2} \mathrm{O},\right)\end{array}$ \\
\hline
\end{tabular}




\begin{tabular}{|c|c|c|}
\hline $\begin{array}{l}\text { Comp. } \\
\text { No }\end{array}$ & IR & NMR. (DMSO) \\
\hline $6 \mathrm{~b}$ & $\begin{array}{l}\text { vNH3131-3410,CH } 3022-2912, \mathrm{vCO} \\
1632 \mathrm{~cm}^{-1} \text { ) and } 1694-1650 \\
\text { corresponding to v CO of carbonyl } \\
\text { pyridazinone moieties. }\end{array}$ & $\begin{array}{l}2.5(\mathrm{~s}, 3 \mathrm{H}, \mathrm{CH} 3), 6.5(\mathrm{~s}, \mathrm{NH}) 6.8-7.9 \\
(\mathrm{~m}, 13 \mathrm{H}, \mathrm{ArH}), 8.2(\mathrm{~s}, 1 \mathrm{H}, \text { proton of } \\
\text { pyridazinone, } 10.2(\mathrm{~s}, 1 \mathrm{H}, \mathrm{NH} \text { acidic } \\
\text { protons }),\end{array}$ \\
\hline $6_{f}$ & $\begin{array}{l}\text { vNH3031-3297), CH 3022-2912, } \\
1694-1650 \mathrm{~cm}^{-1} \text { corresponding to } 2 \mathrm{v} \\
\text { CO of carbonyl of pyrimidinonyl and } \\
\text { pyridazinone moieties }\end{array}$ & $\begin{array}{l}2.3(\mathrm{~s}, 3 \mathrm{H}, \mathrm{CH} 3), 7.0-7.8(\mathrm{~m}, 9 \mathrm{H}, \mathrm{ArH}) \\
8-8.2(\mathrm{~s}, 3 \mathrm{H}, \text { protons of pyridazinone and } \\
\text { pyrimidine moieties, } 10.2(\mathrm{~s}, 1 \mathrm{H}, \mathrm{NH} \text { acidic } \\
\text { protons }), 11.2(\mathrm{~s}, 2 \mathrm{H}, \mathrm{OH})\end{array}$ \\
\hline $7_{\mathrm{a}}$ & $\begin{array}{l}\text { vNH 3031-3297, CH 3022-2912 } \\
\text { v CO } 1741 \mathrm{~cm}^{-1} \text { of ester }\end{array}$ & $\begin{array}{l}\text { 1.3(t,3H,CH3,J=7.4), } 2.5\left(\mathrm{~s}, 3 \mathrm{H}, \mathrm{CH}_{3} \mathrm{CO}\right), \\
3.4\left(\mathrm{q}, 2 \mathrm{H}, \mathrm{CH}_{2-} \mathrm{J}=7.4\right), 4.8(\mathrm{~s}, 2 \mathrm{H}, \mathrm{CH} 26.7 \\
(\mathrm{s}, \mathrm{NH}), 7.0-7.8(\mathrm{~m}, 10 \mathrm{H}, \mathrm{ArH} \text { and proton of } \\
\text { pyridazinone }) 8.2(\mathrm{~s}, 1 \mathrm{H}, \mathrm{COOH}), 8.6 \\
(\mathrm{~s}, 2 \mathrm{H}, \mathrm{NH}-\mathrm{C}=\mathrm{O})\end{array}$ \\
\hline $8 \mathrm{a}$ & $\begin{array}{l}\text { vNH } 3031-3297,3022-2912(\mathrm{C}- \\
\mathrm{H} 1671 \mathrm{~cm}^{-1} \text { corresponding to v CO } \\
\text { of carbonyl of pyridazinone moiety }\end{array}$ & $\begin{array}{l}2.5(\mathrm{~s}, 3 \mathrm{H}, \mathrm{CH} 3 \mathrm{CO}), 5.9-6.5(\mathrm{~m}, 3 \mathrm{H}, \mathrm{NH} 2 \& \\
\left.\mathrm{NH} \text {, disappear in } \mathrm{D}_{2} \mathrm{O}\right), 6.9-7.6(\mathrm{~m}, 9 \mathrm{H}, \\
\text { aromatic protons }), 8.1(\mathrm{~s}, 1 \mathrm{H}, \text { proton of } \\
\text { pyridazine moiety }), 12.5(\mathrm{~s}, 1 \mathrm{H}, \mathrm{CO}-\mathrm{NH}) \\
\text { disappears by } \mathrm{D}_{2} \mathrm{O} .\end{array}$ \\
\hline
\end{tabular}

\section{References}

1.Toshikazu, K., Tukuba Research Lab.Food,Food Ingredients J.Japan, 206 (2002).

2. Barrett, D., Tanaka, A., Harada, K., Ohki, H., Watabe, E., Maki, K. and Ikeda, F., Bioorg. Med. Chem. Lett. 11, 479-482 (2001).

3. Kovalainen, J.T., Christains, J.A.M., Kotisaati, S., Laitinen, J.T. and Mannisto, P.T., J. Med. Chem. 42, 1193 (1999).

4. El-Faham,A., Elmassry, A.M., Amer, A. and Gohar,Y.M., Lett. Pept. Sci. 9, 49 (2002).

5. a) Polyak, F. and Lubell,W.D., J.Org. Chem. 63, 5937 (1998); b) Roy, S., Lombart, H.G., Lubell, W.D., Hancock, R.E.W. and Farmer, S.W., J. Peptide Res. 60, 198 (2002).

6. Okada,Y., Tsukatani, M., Taguchi, H., Yakai,T., Bryant,S.D. and Lazarus, L.H., Chem. Pharm. Bull. 46, 1374 (1998).

7. Sun, G., Uretsky, N.J., Wallace, L.J., Shams, G., Weinstein, D.M. and Miller, D.D., J. Chem. 39, 4430 (1996) .

8. Marsham, P.R., Wardleworth, J.M., Boyle, F.T., Hennequin, L.F., KBrown, M. and Jackman, A.L., Med. Chem. 42, 380 (1999).

9. Xia, Y., Yang, Z.V., Xia, P., Bastow, K.F., Nakanishi, Y. and Lee, K.H., Bioorg. Med. Chem. Lett. 10, 699 (2000).

Egypt. J. Chem. 54, No.3 (2011) 
10. El-Hashash, M.A., Abdel-Mageid, G.A., El-Kady, S.S. and Mohammed, S.A., 1,4Thioarialkylation and/or amination of E-3-(4-bromo benzoyl) acrylic acid and some studies with the products. Egypt. J. Chem. 51(1), 103-112 (2008) .

11. a) Rizk,S.A., El-Hashash, M.A. and Mostafa, K.K., Utility of $\beta$-aroyl acrylic acid in heterocyclic synthesis. Egypt, J. Chem. 51 (5), 116-121 (2008); b) Rizk, S.A., ElHashash, M.A. and Aburzeza, M.M., 1,4-Arylation of $\beta$-(4-acetylaminobenzoyl) acrylic acid with activated aromatic hydrocarbons under Fridel-Crafts conditions and some studies with the products. Egypt. J. Chem. 54 (1), (2011).

12. Xia-Juan Zou, Lu-Hua Lai, Gui-Yu Jin and Zu-Xing Zhang, J. Agric. Food. Chem. 50 (13), (2002).

13. a) Daidone, G., Maggio, B., Plescia, S., Raff, D., Musiu, C., Milia, C., Perra, G. and Marong-iu, M. E., Eur. J. Med. Chem.,33, 375 (1998) ; b) Singh, N., Sangwan, N.K. and Dhindsa, K.S., Pest Manage .Sci. 56, 284 (2000); c) Daidone, G., Raffa, D., Plescia, F., Maggio, B.R. and Occaro, A., Arkivoc (xi) 227 (2002); d) Migliara, O., Plescia, S., Diana, P., Distefano,V., Camarda, L. and Dall, Olio, R., Arkivoc (v),44 (2004); e) Kataritzky, A.R., Vakulenko, A.V., AkueGedu, R., Gromove, A.V., Witek, R. and Rogers, J.W., Arkivoc (i), G. (2007).

14. Kesharwani,K., Sahu, N. K. and Kohli, D.V., J. Pharma. Chem.43, 6 (2009).

15. Jacob, A.M., Thumpakkara, R., Prathapas, S. and Jose, B., Tetrahudron, 61, 4601(2005).

16. Mohamed, M.M., El-Hashash, M.A.I. and Abo-Baker, O.A., Rev. Roum. Chim. 27, 865 (1982).

17. a) Patel, M., Mc-Hvgh, R.J. Jr, Cardova, B.C., Klabe, R.M., Ericksonviitanen, S., Trainor, G.L. and Ko, S.S., Bioorg. Med. Chem. Lett., g, 3221 (1999); b) ELShafei, H.A. and Badr-Eldin, S.M., Egypt. J. Micobiol. 27, 353 (1994); c) Svgimoto, Yon ago, M., Karibe, N., Limure, Y., Nagato, S., Sasaki, A. and Yamanishi, Y., Patent Appl. EP. 468,187 (1992) Chem. A bstr.116, 235643 (1992); d) Waxman, L. and Darke, P.L., Antiviral Chem.ch-emother. 11,1 (2000); e) Girgis, A.S., Pharmazie, 466 (2000).

18. EL-Hashash, M.A. and EL-Nagdy, S.I., J. Chem. Eng. Data 29.

19. Ayed, G.H., Ismail, A.A., Rohat, F., EL-Mobayed, M. and Mohamed, S.M., Comac. Sci. Univ, Ankarar, B. Chem. Eng. 36, 53 (1990), Chem. Abst, 123, 25662 SV (1995).

(Received 4/1/2011; accepted 8/ 1/2012) 

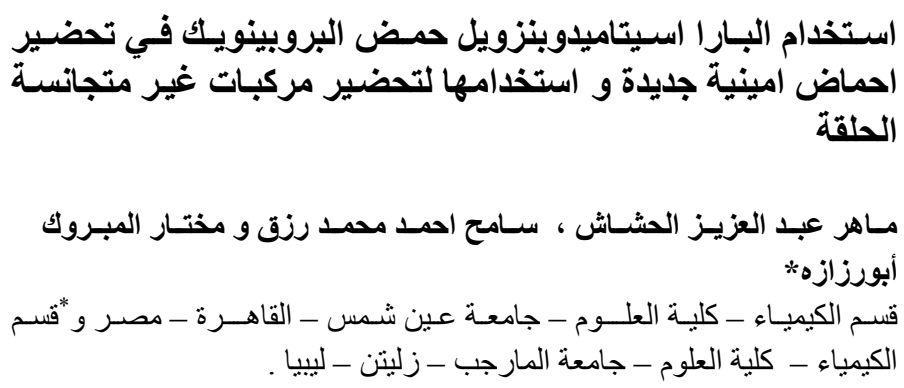

يتضمن هذا البحث 1ـ تحضير بعض الاحمـاض الامينية الصناعية التى لها نشـاط

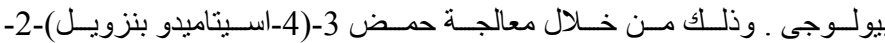

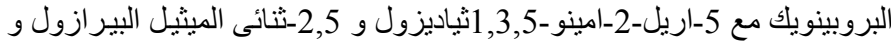

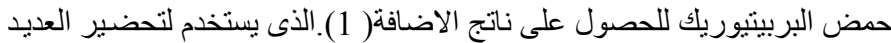

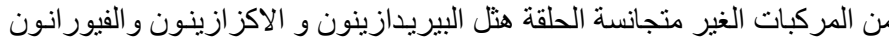

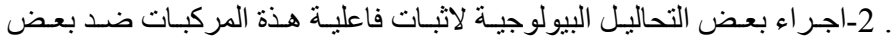

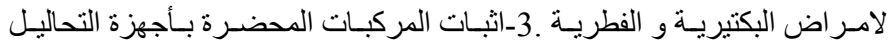
الدقيقة مثل الاشعة تحت الحمر اء و الرنين المغناطيسى والكتلة الاكترونى. 